\title{
Complex Cardiovascular Surgery in Jehovah's Witness Patients
}

E Ramiaramanana ${ }^{1}$, S Taamalah ${ }^{1}$, N Rabah ${ }^{1}$, A Al Karaky ${ }^{3}$, Imen Benhadj ${ }^{4}$ and C Vaislic ${ }^{2}$

${ }^{1}$ Eure-Seine Intercity Hospital, Vernon, Le Chesnay, France

${ }^{2}$ Parly II Medico-Surgical Centre, Le Chesnay, France

${ }^{3}$ Nevers Hospital, France

${ }^{4}$ Valenciennes Hospital, France

\begin{abstract}
Background: Cardiovascular surgery in patients who are Jehovah's Witnesses (JW) poses both technical and ethical challenges because these patients strictly refuse the administration of allogeneic blood. The challenge is particularly great in patients who have co-morbidities or who undergo complex procedures.

Methods: We retrospectively reviewed the characteristics and outcomes of 153 consecutive JW patients who underwent cardiovascular surgical procedures between January 2005 and January 2013 at the Parly II MedicoSurgical Centre in Le Chesnay, France. Our first objective was to compare perioperative outcomes (morbidity and mortality) between a group of patients with co-morbidities posing specific challenges in terms of transfusion avoidance and the general JW population operated at our institution during the same period. Our second objective was to compare morbidity and mortality outcomes between patients undergoing simple procedures and those undergoing complex or combined procedures.

Results: Of $153 \mathrm{JW}$ patients operated during the study period, 13 (8.5\%) had co-morbidities posing specific challenges in terms of transfusion avoidance and were classified as high-risk patients. Among high-risk patients, mean EuroSCORE was higher $(5.23$ (range, 0-14) vs. 4.37 (range, $0-16.76$ ); $p=0.005$ ) and there was a greater proportion of redo procedures ( $23 \%$ vs. $11.4 \%$; $p=0.001)$. Compared with the control group, the high-risk group had a 2.4 -fold longer mean ICU length of stay ( 6.5 vs. 2.7 days; $p=0.001)$ and a higher perioperative mortality $(23 \%$ vs. $2.1 \% ; p=0.001)$. Forty-nine patients $(32 \%)$ underwent complex procedures, whereas 104 patients $(68 \%)$ underwent simple procedures involving a single surgical repair. The perioperative complication rate was $20.4 \%$ among patients undergoing complex procedures, compared with $11.5 \%$ in the control group $(p=0.001)$. Perioperative mortality was $4.08 \%$ in the group undergoing complex procedures, compared with $3.85 \%$ among patients requiring single-repair procedures $(p=0.005)$. Among patients undergoing redo surgery, mortality rates were $11.1 \%$ and $2 \%$ in patients who underwent complex and simple procedures, respectively $(p=0.001)$, whereas in patients with a first surgery the corresponding mortality rates were $2.5 \%$ and $2.1 \%$, respectively $(p=0.005)$.
\end{abstract}

Discussion: In our experience, complex cardiovascular surgical procedures can be performed with good outcomes in JW patients who have risk factors in addition to their main surgical pathology or require complex procedures.

Keywords: Cardiovascular surgery; Blood conservation; Multidisciplinary approach; Jehovah's witnesses; Perioperative outcomes

\section{Background}

Cardiovascular surgery in patients who are Jehovah's Witnesses (JW) poses both technical and ethical challenges because these patients strictly refuse the administration of allogeneic blood (red blood cells, plasma and platelets). The challenge is particularly great in patients who have co-morbidities or who undergo complex procedures, the majority of whom would typically be transfused. Avoiding the use of allogeneic blood transfusion in this context requires a global, multidisciplinary team approach. We have previously reported on the development of a blood-saving protocol $[1,2]$ and its use in 500 Jehovah's Witnesses patients [3].

In this retrospective, observational, case-control study, we compared a group of JW patients presenting with co-morbidities that posed specific challenges in terms of transfusion avoidance with the general population of JW patients operated at our institution during the same period. We also compared the outcomes of JW patients who underwent relatively simple cardiac procedures involving a single surgical repair with those of JW patients who required complex surgical procedures involving multiple defects. Our objective was to show that complex cardiovascular surgical procedures can be performed with good outcomes in JW patients who have risk factors in addition to their main surgical pathology or require complex procedures.

\section{Methods}

We retrospectively reviewed the characteristics and outcomes of 153 consecutive JW patients who underwent cardiovascular surgical procedures between January 2005 and January 2013 at the Parly II Medico-Surgical Centre in Le Chesnay, France. Consultation with local ethics committee established inclusion and exclusion criteria, thus we excluded emergency procedures, congenital heart disease, cardiogenic shock, patients in whom a prolonged EPO treatment did not produce a hemoglobin level of $14 \mathrm{gm}$, patients with problems of

*Corresponding author: Claude D Vaislic, Consultant, CHP PARLY 2, Cardiac Surgery, 21 RUE, Moxouris, Le Chesnay, 78140, France, Tel: 0033663294841; E-mail: claudevaislic@hotmail.com

Received May 21, 2015; Accepted June 09, 2015; Published June 15, 2015

Citation: Ramiaramanana E, Taamalah S, Rabah N, Karaky AA, Benhadj I, et al. (2015) Complex Cardiovascular Surgery in Jehovah's Witness Patients. J Vasc Med Surg 3: 205. doi:10.4172/2329-6925.1000205

Copyright: (C) 2015 Ramiaramanana E, et al. This is an open-access article distributed under the terms of the Creative Commons Attribution License, which permits unrestricted use, distribution, and reproduction in any medium, provided the original author and source are credited. 
hemostasis or those in whom coagulopathy was anticipated following the implementation of extra-corporal circulation and patients with thoraco-abdominal aneurysms in which we could not reasonably avoid transfusion. Two patients suffering from aortic dissection were excluded due to initial preoperative hemoglobin levels inferior to 15 g/L. Two patients could not be operated upon due to the impossibility of attaining sufficient levels of hemoglobin prior to the scheduled procedure, despite a month of EPO treatment. One of these patients showed signs of an increase in the size of a type 1 aortic dissection which became chronic following his refusal of a transfusion despite the advice of the caring team. The other patient could not be operated upon due to a reaction to EPO of unidentifiable cause. From 2005 onwards the use of mini-cardiopulmonary bypass circuit, retrograde autologous priming and recombinant human erythropoietin (rHuEPO) allowed us to perform more complex cases. However, during this period we had to stop using the antifibrinolytic drug aprotinin to decrease perioperative blood loss because the drug was withdrawn from the market.

Our first objective was to compare perioperative outcomes (morbidity and mortality) in a group of patients with co-morbidities and the general JW population undergoing cardiovascular surgery at our institution. The following parameters were considered: 1) age; 2) European system for cardiac operative risk evaluation (EuroSCORE); 3) preoperative haemoglobin concentration; 4) number of redo procedures in each group; 5) Intensive Care Unit (ICU) length of stay; and 6) perioperative mortality.

Our second objective was to compare morbidity and mortality outcomes in patients undergoing simple procedures and in those undergoing complex or combined procedures. In order to do so, we divided this cohort into two groups: patients requiring a single surgical repair and patients in whom more than one repair was performed. For this comparison, we analysed 1) the complication rate and 2) mortality in each group.

\section{Statistical Analysis}

The hypothesized normality of the distribution of continuous variables was verified by a Kolmogorov-Smirnoff test. The continuous variables are expressed as means of standard deviation and the differences were evaluated by Student $T$ test. Variable analysis was conducted by Chi-Square or Fisher tests according to the size of the sample. Statistical significance was presumed when the $\mathrm{P}$ value was found to be less than 0.05. Stataxt software package (Cytel Software Corporation, Cambridge, Mass.) was used in the statistical analysis.

\section{Results}

\section{First comparison: patients with co-morbidities versus lower-} risk patients

A total of $153 \mathrm{JW}$ patients underwent cardiovascular surgical procedures at our institution between January 2005 and January 2013. Of these, $13(8.5 \%)$ had co-morbidities posing specific challenges in terms of transfusion avoidance (Table 1) and were classified as highrisk patients, while the remaining 140 patients were considered at lower risk

Mean age was 62.5 years (range, 37-83) in the high-risk group compared with 66.6 years (range, 28-91) in the low-risk group $(\mathrm{p}=0.005)$. Table 2 shows the age distribution in each group.

The EuroSCORE was available for all except one patient. Mean EuroSCORE was 5.23 (range, 0-14) in the high-risk group compared with 4.37 (range, $0-16.76)$ in the control group ( $\mathrm{p}=0.005)$.
Mean preoperative haemoglobin concentration was similar in both groups: $14.5 \mathrm{~g} / \mathrm{dL}$ (range, 13.1-15.9) in patients with co-morbidities and $14.46 \mathrm{~g} / \mathrm{dL}$ (range, 11-17) in low-risk patients ( $\mathrm{p}=0.005)$.

Three patients (23\%) in the group with comorbidities versus 16 patients in the control group (11.4\%) underwent redo procedures $(\mathrm{p}=0.005)$.

Patients in the high-risk group had a 2.4-fold longer mean ICU length of stay ( 6.5 vs. 2.7 days; $(p=0.001)$,) than lower-risk patients. The longest ICU stay was 45 days in the high-risk group compared with 20 days in the control group.

Six of 153 patients $(3.9 \%)$ died in the perioperative period. Perioperative mortality was significantly higher in the high-risk group ( 3 of $13 ; 23 \%)$ than in the low-risk group ( 3 of $140 ; 2.1 \%)(p=0.001)$.

\section{Second comparison: complex versus simple procedures}

Of the $153 \mathrm{JW}$ patients who were operated at our institution between January 2005 and January 2013, 49 (32\%) underwent procedures involving more than one surgical repair (complex procedures), whereas 104 patients $(68 \%)$ underwent simple procedures involving a single surgical repair.

The perioperative complication rate was $20.4 \%$ among patients undergoing complex procedures, compared with $11.5 \%$ in the control group $(\mathrm{p}=0.001)$.

The perioperative mortality rate was $4.08 \%$ in the group undergoing complex procedures, compared with $3.85 \%$ among patients requiring single-repair procedures ( $\mathrm{p}=0.001)$, Among patients with redo surgery, mortality rates were $11.1 \%$ and $2 \%$ in patients who underwent complex and simple procedures, respectively $(\mathrm{p}=0.001)$, whereas in patients with a first surgery the corresponding mortality rates were $2.5 \%$ and $2.1 \%$, respectively $(\mathrm{p}=0.005)$.

\section{Preoperative administration of recombinant human erythropoietin}

Preoperative rHuEPO administration was used in $54.4 \%$ of

\begin{tabular}{|l|c|}
\hline Co-morbidities & Number of patients \\
\hline Haemorrhagic colon cancer & 1 \\
\hline Sickle-cell disease & 1 \\
\hline Endocarditis & 2 \\
\hline Left ventricular ejection fraction =10\% & 1 \\
\hline Myocardial infarction & 1 \\
\hline Renal failure / dialysis & 2 \\
\hline Chronic myeloid leukaemia & 1 \\
\hline Myasthenia gravis & 1 \\
\hline Immune system disease (hydroxychloroquine treatment) & 1 \\
\hline Takayasu's arteritis & 1 \\
\hline Thalassaemia & 1 \\
\hline Total & $\mathbf{1 3}$ \\
\hline
\end{tabular}

Table 1: Patients' co-morbidities in the high-risk group.

\begin{tabular}{|c|c|c|}
\hline Age (years) & High-risk group (N) & Low-risk group (N) \\
\hline$\leq 30$ & 0 & 1 \\
\hline$>30-50$ & 2 & 15 \\
\hline$>50-70$ & 7 & 65 \\
\hline$>70-90$ & 4 & 58 \\
\hline$>90-99$ & 0 & 1 \\
\hline Total & $\mathbf{1 3}$ & $\mathbf{1 4 0}$ \\
\hline
\end{tabular}

Table 2: Age distribution in the high- and low-risk groups. 
the patients. The proportion of patients treated with rHuEPO was higher in the group undergoing simple procedures than in the group undergoing complex procedures $(68.7 \%$ vs. $31.2 \%$; $(\mathrm{p}=0.001)$. While $85 \%$ of rHuEPO-treated patients were in the lower-risk group, $85.7 \%$ of patients with risk factors received $\mathrm{rHuEPO}$ in the preoperative period. Among rHuEPO-treated patients, 3.75\% died perioperatively. Of the 6 patients who died, 3 had received $\mathrm{rHuEPO}$.

\section{Discussion}

The lower than expected mortality here reported is explained by very strict inclusion criteria, including a preopertative level of haemoglobin greater than $14 \mathrm{~g} / \mathrm{dL}$, delaying surgery to wean the patient of thrombolytics, antiplatelets and anticoagulant agents. Given these limits, the results of this observational study show that, complex cardiovascular surgery can be performed without blood transfusion. However, this can only be achieved by strictly applying a multidisciplinary blood-conservation protocol. First, we aim for a preoperative haemoglobin level in the range of $14-15 \mathrm{~g} / \mathrm{dL}$. Since the risk of intraoperative bleeding complications cannot be eliminated, this pre-required condition is essential [4]. In case of complex procedures, preoperative stimulation of erythropoiesis with rHuEPO plays an important role in these patients who refuse blood transfusion.

This decision has important implications. Firstly, weleave the patient with an untreated cardiac pathology for one or several months until the red cell mass had increased as a result of rHuEPO administration. Two patients could not be operated because they did not respond to rHuEPO therapy, and two patients died from haematological causes while on the waiting list. This clearly represents a loss of chance. Secondly, some pathologies, namely haemolytic anaemias and leukaemia, confronted us with ethical dilemmas. Only after taking advice from a multidisciplinary team including haematologists did we decide to use rHuEPO in two patients with sickle-cell disease and thalassaemia, respectively, with the risk of stimulating the production of abnormal haemoglobin, and in one patient with leukaemia, with the risk of inducing disease progression. Three and five years after surgery, respectively, these three patients are alive and asymptomatic.

In patients with sickle cell anaemia, red blood cell transfusion is usually indicated at haemoglobin concentrations of $8 \mathrm{~g} / \mathrm{dL}$ or below. Ideally, red blood cells are administered immediately or up to two weeks before the surgical procedure, with a target haemoglobin concentration of $10 \mathrm{~g} / \mathrm{dL}$, which suppresses erythropoiesis and decreases the proportion of sickle red blood cells $[5,6]$. Therefore, preoperative $\mathrm{rHuEPO}$ administration would appear contradictory in this setting. In our cohort, the only patient with sickle cell disease was treated with rHuEPO preoperatively before an aortic valve replacement and had no particular complications. The patient's haemoglobin concentration before surgery was $14.1 \mathrm{~g} / \mathrm{dL}$.

Unlike sickle cell disease, thalassaemia is a good indication for rHuEPO administration before a high-blood-loss surgical procedure associated. Preoperative use of rHuEPO combined with autologous blood donation was reported in a patient with thalassaemia minor undergoing scoliosis repair [7]. The only patient with thalassaemia in our case series did not receive rHuEPO; surgery was performed successfully and the patient did not experience any complications.

One patient had chronic myeloid leukaemia. Current practice guidelines recommend the use of Erythropoiesis-Stimulating Agents (ESAs) in patients with myelodysplatic syndromes to treat anaemia and decrease the need for blood transfusion $[8,9]$. Our patient was successfully treated with $\mathrm{rHuEPO}$ and had a preoperative haemoglobin concentration of $15.1 \mathrm{~g} / \mathrm{dL}$.

In patients with end-stage heart failure, some authors report the feasibility of complex cardiovascular surgical procedures such as heart transplantations, with comparable outcomes in JW patients as those accepting blood transfusions [10]. In our series, a patient with a left ventricular ejection fraction below $10 \%$ and a preoperative haemoglobin concentration of $13.5 \mathrm{~g} / \mathrm{dL}$ underwent aortic valve replacement surgery without complications.

When a multimodal blood conservation protocol is used, JW patients who undergo major cardiovascular surgery do not appear to be at increased risk of surgical complications or long-term mortality than patients who accept blood transfusion [11].

In our experience, complex cardiovascular surgical procedures can be performed with good outcomes in JW patients who have risk factors in addition to their main surgical pathology or require complex procedures.

\section{References}

1. Vaislic C, Bical O, Farge C, Gaillard D, Ponzio O, et al. (2003) Totally minimized extracorporeal circulation: an important benefit for coronary artery bypass grafting in Jehovah's witnesses. Heart Surg Forum 6: 307-310.

2. Vaislic C, Bical O, Deleuze P, Khoury W, Gaillard D, et al. (2005) Cardiac surgery without transfusion in 2005. Arch Mal Coeur Vaiss 98: 7-12.

3. Vaislic CD, Dalibon N, Ponzio O, Ba M, Jugan E, et al. (2012) Outcomes in cardiac surgery in 500 consecutive Jehovah's Witness patients: 21 year experience. J Cardiothorac Surg 7: 95.

4. Viele MK, Weiskopf RB (1994) What can we learn about the need for transfusion from patients who refuse blood? The experience with Jehovah's Witnesses. Transfusion 34: 396-401.

5. Hirst C, Williamson L (2012) Preoperative blood transfusions for sickle cell disease. Cochrane Database Syst Rev 1.

6. Howard J, Malfroy M, Llewelyn C, Choo L, Hodge R, et al. (2013) The Transfusion Alternatives Preoperatively in Sickle Cell Disease (TAPS) study: a randomised, controlled, multicentre clinical trial. See comment in PubMed Commons below Lancet 381: 930-938.

7. Perez Ferrer A, Ferrazza V, Gredilla E, de Vicente J, de la Rua A et al (2007) Bloodless surgery in a patient with thalassemia minor. Usefulness of erythropoietin, preoperative blood donation and intraoperative blood salvage. Minerva Anestesiol 73: 323-326.

8. Rizzo JD, Brouwers M, Hurley P, Seidenfeld J, Somerfield MR, et al. (2010) American Society of Clinical Oncology/American Society of Hematology clinical practice guideline update on the use of epoetin and darbepoetin in adult patients with cancer. J Clin Oncol 28: 4996-5010.

9. Greenberg PL, Attar E, Bennett JM, Bloomfield CD, Borate U, et al. (2013) Myelodysplastic syndromes: clinical practice guidelines in oncology. $\mathrm{J}$ Nat Compr Canc Netw 11: 838-874.

10. Elmistekawy E, Mesana TG, Ruel M (2012) Should Jehovah's Witness patients be listed for heart transplantation? Interact Cardiovasc Thorac Surg 15: 716719 .

11. Pattakos G, Koch CG, Brizzio ME, Batizy LH, Sabik JF 3rd, et al. (2012) Outcome of patients who refuse transfusion after cardiac surgery: a natura experiment with severe blood conservation. Arch Intern Med 172: 1154-1160. 